\title{
Response of Predatory Mites to a Herbivore-Induced Plant Volatile: Genetic Variation for Context-Dependent Behaviour
}

\author{
Beata Sznajder • Maurice W. Sabelis • Martijn Egas
}

Received: 31 December 2009 / Revised: 31 May 2010 /Accepted: 7 June 2010 / Published online: 25 June 2010

(C) The Author(s) 2010. This article is published with open access at Springerlink.com

\begin{abstract}
Plants infested with herbivores release specific volatile compounds that are known to recruit natural enemies. The response of natural enemies to these volatiles may be either learned or genetically determined. We asked whether there is genetic variation in the response of the predatory mite Phytoseiulus persimilis to methyl salicylate (MeSa). MeSa is a volatile compound consistently produced by plants being attacked by the two-spotted spider mite, the prey of $P$. persimilis. We predicted that predators express genetically determined responses during longdistance migration where previously learned associations may have less value. Additionally, we asked whether these responses depend on odors from uninfested plants as a background to MeSa. To infer a genetic basis, we analyzed the variation in response to MeSa among iso-female lines of P. persimilis by using choice-tests that involved either (1) MeSa presented as a single compound or (2) MeSa with background-odor from uninfested lima bean plants. These tests were conducted for starved and satiated predators, i.e., two physiological states, one that approximates migration and another that mimics local patch exploration. We found variation among iso-female lines in the responses to MeSa, thus showing genetic variation for this behavior. The variation was more pronounced in the starved predators, thus indicating that $P$. persimilis relies on innate preferences when migrating. Background volatiles of uninfested plants changed the predators' responses to $\mathrm{MeSa}$ in a manner that depended on physiological state and iso-female line. Thus,
\end{abstract}

\footnotetext{
B. Sznajder $(\triangle) \cdot M$. W. Sabelis $\cdot$ M. Egas

Department of Population Biology, Institute for Biodiversity and Ecosystems Dynamics, University of Amsterdam, Science Park 904, P.O.Box 94248, 1090 GE Amsterdam, the Netherlands

e-mail: b.a.sznajder@gmail.com
}

it is possible to select for context-dependent behavioral responses of natural enemies to plant volatiles.

Key Words Phytoseiulus persimilis · Methyl salicylate . I-tube olfactometer Preference $\cdot$ Genetic variation . Context dependence

\section{Introduction}

Predatory arthropods are known to respond to herbivoreinduced plant volatile chemicals (Dicke and Van Loon 2000; Sabelis et al. 2007). This phenomenon prompted the hypothesis that first and third trophic levels "conspire" against the second: the infested plants attract the natural enemies that can reduce or eliminate herbivore pressure, whereas the predators acquire information on the location of its prey (Dicke and Sabelis 1988; Dicke and Van Loon 2000; Kessler and Baldwin 2001, but see Allison and Hare 2009). However, for such a system to evolve and function, a number of conditions have to be fulfilled (Van der Meijden and Klinkhamer 2000; Janssen et al. 2002). Among them, it requires that the predators evolve behavioral responses to plant-produced volatiles induced by herbivore feeding. These responses may rely on predators learning to associate herbivore-induced plant volatiles with the presence of prey. Alternatively, predators evolved genetically determined preferences for plant volatiles induced by herbivorous prey, if in the past generations predators innately responding to such volatiles (i.e., prior to any experience) had higher fitness than those that did not show such behavior. Fitness benefit would be obtained because these volatiles were reliably coupled with the presence of prey. Therefore, we expect genetic bases for predator responses to these plant volatiles that are induced 
by the feeding of prey, irrespective of the species of infested plant.

We investigated whether there is genetic variation in the response of the predatory mite Phytoseiulus persimilis (Athias-Henriot) to a selected herbivore-induced plant volatile, methyl salycilate (MeSa). MeSa is one of the volatile compounds consistently induced by feeding of the twospotted spider mite (Tetranychus urticae Koch) - the prey of $P$. persimilis - on a variety of plant species (van den Boom et al. 2004). Empirical studies showed that it plays a key role in predator attraction to the volatile blends induced by the spider mite (de Boer and Dicke 2004a; de Boer et al. 2004; van Wijk et al. 2008; Ament et al. 2010). A genetic basis for the responses of $P$. persimilis to blends of volatile compounds has been demonstrated in two studies on selection for responses of satiated predators to the complete blend of volatiles released by spider-mite infested lima bean (Margolies et al. 1997; Jia et al. 2002). However, innate preferences for the full volatile blend of infested bean may be the result of the underlying innate response to a single compound shared by the blends of many plant species infested by the spider mite - a condition that is fulfilled by MeSa. In this study, we applied an analysis using iso-female lines (David et al. 2005) to detect genetic variation in response to MeSa among $P$. persimilis lines. A significant difference in the olfactory responses among iso-female lines indicates a genetic basis for this trait, provided that systematic environmental influences are controlled for.

The odor of uninfested plants is a permanent feature of the volatile signal under natural settings. Therefore, we hypothesized that the background odors of uninfested lima bean may affect the predator's perception of MeSa and the strength of response to MeSa. Lima bean leaves heavily infested with spider mites also were used for culturing of the predator population used in this experiment. Therefore, any pre-conditioning of the predator to the odors experienced in the culture would be to the volatile blend of infested lima bean that is quantitatively different from the blend of uninfested bean (e.g., Dicke et al. 1990). Hence we expected that an enhanced predator response to $\mathrm{MeSa}$ presented with the background odors of uninfested lima bean would be a result of altered perception to MeSa when presented within a context rather then due to predator preconditioning to specific volatiles. Mechanically damaged plants produce increased amounts of a distinct group of volatiles called green leaf volatiles (GLVs), which are also emitted by intact plants in smaller amounts (Hatanaka 1993; Matsui et al. 2000; van den Boom et al. 2004). Thus, we term the background odors provided by the punched leaf discs used in this experiment as GLVs.

Genetically determined responses to herbivore-induced plant volatiles are particularly relevant, and subject to natural selection, in situations where predators cannot yet have learned the association between the presence of specific volatiles and prey. The predatory mite $P$. persimilis may experience such situations during the migratory phase that follows the exploitation of the previous colony of prey. These phases of the predator life history can be approximated by manipulating the predator's hunger level because food conditions provide the proximate cue for the onset of migratory behavior (Sabelis and Afman 1994). Satiation prevails during foraging in dense colonies of spider mites, and starvation induces take-off to aerial dispersal, followed by exploration of the new landing site (Sabelis and van de Baan 1983; Sabelis et al. 1984; Sabelis and van der Meer 1986; Sabelis and van der Weel 1993; Sabelis and Afman 1994; Pels and Sabelis 1999). During the migratory phase, the predators encounter an environment characterized by plant volatiles most likely to be different from those experienced before; the previously learned associations of specific volatiles may be of little value in locating the prey. Therefore, we hypothesized that the innate responses to MeSa depend on the context of the test (satiated vs. starved predators).

We determined the responses of a total of 18 iso-female lines of $P$. persimilis in the olfactory tests where predators were presented with the choice of 1) MeSa in clean air vs. clean air, or 2) MeSa in the background of GLVs vs. GLVs. These tests were performed using either satiated or starved predators. Variable olfactory responses to MeSa among the iso-female lines would indicate genetic variation for this trait in the population under study. In particular, we predicted that this variation would be more pronounced in starved predators, and we asked to what extent this variation depends on the presence of volatiles of uninfested plants in the background.

\section{Methods and Materials}

Predatory Mites The base population of $P$. persimilis was maintained in the laboratory $\left(25^{\circ} \mathrm{C}, 70 \%\right.$ humidity, and 16:8, L:D conditions) on a diet of two-spotted spider mites (T. urticae) on detached leaves of lima bean plants. The culture originated from a sample of predators collected in 2002 at different locations throughout Sicily (Partinico, Scopella, Trappeto, Terrasini, Siculiana, Laghetto, Menfi, Trabia, Alcamo), where they are probably endemic (De Moraes et al. 2004). Iso-female lines were obtained by randomly selecting a number of females from the base population, which from then on were kept separately in Petri dishes. Typically in haplodiploid arthropods such lines are obtained through mating between the virgin female and her sons (oedipal mating). However, P. persimilis, just as probably the whole family Phytoseiidae, is pseudoarrhenotokous, i.e., sons and daughters arise from fertilized 
eggs, and male zygotes become haploid due to paternal genome inactivation and elimination during embryogenesis (Helle et al. 1978; Nelson-Rees et al. 1980). Hence, mating is a prerequisite for producing eggs destined to become females, as well as males. For this reason, we selected already mated females, and propagation of the lines occurred through brother-sister mating. There were 18 lines established, which were allowed to propagate for $7 \mathrm{wk}$ before the onset of the olfactory tests (for at least 5 generations). All lines were reared in the same climate room (under conditions as described for the base population) to minimize any effect of environment on predatory behavior. Incidental mixing among the lines was prevented by maintaining each line in a separate rearing enclosed in a plastic container with a small opening covered with gauze (mesh width $=0.07 \mathrm{~mm}$, average adult predator size $=$ $0.5 \mathrm{~mm}$, average predator egg size $=0.2 \mathrm{~mm}$, small enough to prevent immi- and emigration) to allow for airflow. Additionally, each of these containers was surrounded by its own water barrier. Individual iso-female lines were labelled with numbers (1-18) for identification.

Tests of Olfactory Preference Behavioral responses of predators to MeSa were tested in a so-called I-tube: a single straight glass tube (length $20 \mathrm{~cm}, 0.5 \mathrm{~cm}$ diam) with a small opening $(2 \mathrm{~mm})$ in the middle to introduce the predatory mites (Fig. 1). This olfactometer was provided with a trap at the end of either arm of the I-tube to collect predators. Opposite the entrance hole, another opening $(0.5 \mathrm{~cm}$ diam) was present that was gauze-covered and served as an air-outlet. At the ends of the I-tube, there were plastic trap vials (30 $\mathrm{mm}$ diam, height $55 \mathrm{~mm}$ ), which in turn were connected to jars that either contained a capillary with MeSa or not. The traps were designed as an easy-to-enter-yet-difficult-to-exit vial, and were provided with a water source (wet cotton wool). After purification by activated carbon filters, the air was flowing at $20 \mathrm{l} / \mathrm{h}$ through the jars, the vials, and then the arms of the glass tube, leaving the system through the opening in the middle of the I-tube. At release in the middle of the I-tube, a mite found itself in air streams coming from right and left, i.e., one with $\mathrm{MeSa}$ and the other without. Subsequently, it could move left or right in the I-tube and ultimately entered one of the trap vials or remained in the Itube. After release of the test animals, the entrance hole was sealed with Parafilm ${ }^{\circledR}$. Pilot experiments showed that the Itube olfactometer produced results consistent with our knowledge of the responses of $P$. persimilis to the full blend of herbivore-induced plant volatiles (Van Wijk, unpublished data).

Per replicate experiment, a total of 25 adult females were released (except for a few cases where numbers were less than 25 , yet larger than 20) sequentially. Visual cues play no role in predator orientation, as the predator is blind and

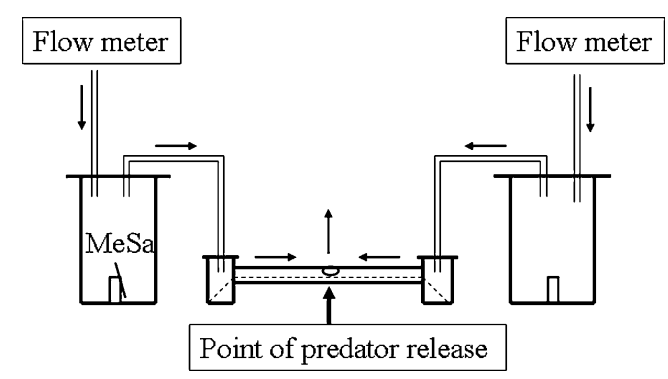

Fig. 1 Experimental set-up of the I-tube olfactometer. The diagram depicts a horizontal tube (the glass I-tube) with two openings, where the bigger one serves as an air-outlet. The thick vertical arrow below the I-tube indicates the location of the small opening-the point of release of the predators. The broken line indicates a thin capillary inside the glass tube to provide structure, on each side ending in a metal pin leading down into the trap vial. The trap vials were connected with plastic tubes to jars that either contain a capillary with MeSa or not. The remaining arrows indicate the direction of air-flow. Above the set-up there was a source of dispersed light (not shown)

orients itself by a means of chemical cues. A previous study that used a Y-tube set-up showed that there is no effect of possible residues, deposited along the path taken by an individual, on the choices of the subsequent individuals (Sabelis and van de Baan 1983). After 25 min, the number of mites in each of the two trap vials was counted. The number that remained in the I-tube, was scored as "no choice". For each consecutive replicate of a line, a new clean I-tube was used, and the side of the arm containing air with $\mathrm{MeSa}$ was inter-changed to exclude any unforeseen asymmetries in the experimental set-up. Per line per treatment, roughly 120 mites were tested in $4-8$ replicate experiments (in one case, three replicates were performed).

Synthetic MeSa (Sigma-Aldrich Fluka, pure; assay $\geq 99 \%$ ) was offered undiluted in a small capillary $(9 \mu \mathrm{l}, 0.60 \mathrm{~mm}$ diam, Omnilabo) placed in one of the jars connected to the Itube (Fig. 1). The air flow was led through the set-up at least $2 \mathrm{~h}$ before the start of the test. The MeSa evaporation rate was $\pm 30 \mu \mathrm{g} / \mathrm{h}$. This evaporation rate was chosen based on preliminary experiments to find a concentration to which the base population exhibited a neutral (50:50) response. Such a set-up allows detection of either increased or decreased responses of the iso-female lines to MeSa with reference to the response of the base population. It is difficult to compare this evaporation rate with previous studies that attempted to measure the dose-response relations, because these assessments were based on filter paper as a substrate (de Boer and Dicke 2004b; van Wijk et al. 2008). Unlike filter paper, capillaries generate a constant evaporation rate. It is also difficult to compare this evaporation rate with evaporation from infested Lima bean plants, because there are hardly any data available. The only data we are aware of is $0.4-0.8 \mu \mathrm{g} /$ h estimated by de Boer and Dicke (2004b) from data in Dicke et al. 1999, evaporated from 10 leaves after $3 \mathrm{~d}$ of infestation 
by 50 adult female spider mites per leaf with a flow rate of $30 \mathrm{l} / \mathrm{h}$. It is unclear at what distance from the plant this concentration was measured, and also how such concentrations of MeSa changed over the time of infestation.

Experimental Design We assessed olfactory preferences of 18 iso-female lines by subjecting satiated and starved adult female predators to two types of olfactory tests that involved choices between either (1) MeSa in clean vs. clean air, or (2) MeSa with a background of green leaf volatiles (GLVs) vs. green leaf volatiles (GLVs). The background odors of GLVs were provided by discs $(1.5 \mathrm{~cm} \mathrm{diam})$ punched from the leaves of 2-wk-old, uninfested Lima bean plants (var. Phaseolus lunatus, Big Lima). New leaf discs were punched for each test from fresh primary leaves, and placed inside the plastic trap vials of the Itube - one disc in each of the vials - on a ball of wet cotton wool. Airflow was allowed to pass through the I-tube for an additional $15 \mathrm{~min}$ before the start of the olfactory test to allow the GLVs to reach the middle of the I-tube. The responses of the satiated predators were determined by testing females taken straight from the culture where they were kept in a well fed state. Starved predators were obtained by food-deprivation for $24 \mathrm{~h}$ prior to the olfactory tests. During the period of food deprivation, mites were kept in a closed Eppendorf vial placed in a climate box at $18^{\circ} \mathrm{C}$, with water provided via a strip of wet filter paper.

The total of 18 olfactory tests performed daily on each of the 18 lines was about the maximal number feasible under the experimental protocol. The main aim of this study was to sample a large number of iso-female lines. Therefore, we preferred to test as many lines as possible per day, at the expense of obtaining simultaneous replicates for each line. To minimize effects of the time of day on predatory responses, we randomized the order of testing of the 18 lines each day. The number of replicates varied between four and six per hunger level for each olfactory test (i.e., tests with or without GLVs context). Tests were performed within a period of $5 \mathrm{wk}$.

Statistical Analysis We aimed to determine (1) whether there is a genetic component in predator response to $\mathrm{MeSa}$, and (2) whether this behavior is dependent on the nutritional status or the environmental context provided by the volatiles of uninfested plants in the background. To address the first question we constructed a mixed-effects model that included the iso-female line effect as the random effect; hence, the response variable (i.e., a single data point) was the mean response per replicate per line based on the numbers of individuals that made a choice (hence, excluding the no-choice individuals). In this analysis, we tested whether the amount of variation explained by the random effect of iso-female line is different from zero. The variation due to iso-female line is a measure of the total amount of variation among tested lines that is due to variation in their genes, provided that the differences among the lines arising from heterogeneity in rearing conditions can be excluded (David et al. 2005). It reflects broad-sense heritability that comprises the additive genetic variation as well as the non-additive effects of dominance and epistasis. The amount of this variation was estimated by using restricted-maximum likelihood method of the SPlus 6.2 software (Pinheiro and Bates 2000; Venables and Ripley 2002), which adjusted for the fixed effects of hunger level (satiated or starved) and GLVs context (MeSa presented alone or MeSa in the background of GLVs). Additionally, we tested whether the responses of the lines changed within the period of testing such that there was a decreasing or increasing trend. To this end we also tested the covariate representing the number of days elapsed from the onset of testing the first replicate of a line within a treatment. A significant covariate would indicate that environmental effects, consistent in time, contribute to the differences among the lines. A non-significant covariate, on the other hand, would indicate that no effect of consistent differences among the rearing environment of the lines was detected, thus strengthening the argument that the variation among the lines reflects genetic differences. The number of days elapsed since the onset of testing encompassed a period of 1-2 wk. Thus, it may have encompassed the responses of more than one generation of predators (Sabelis 1981). A non-significant covariate would be consistent with a positive correlation between mean values in different generations (a result that supports a genetic basis for preference within iso-female lines, David et al. 2005), although the design of this study did not address this correlation explicitly. The diagnostics plots showed that the assumptions of the mixed effect model were satisfied (the random variables were normally distribution with mean zero and independent for different groups, and the withingroup errors were independent and normally distributed with a mean of zero), hence, the response variable was not transformed.

To address the second question we tested for the situation where different genotypes respond to $\mathrm{MeSa}$ differently, dependent on predator satiation level or volatile presentation (as reflected in two- and three-way interactions between the line effect and the experimental factors). Therefore, in the second part of the analysis, we also analyzed the probability of an individual choosing MeSa in relation to three explanatory variables: iso-female line (18 lines), hunger level (satiated or starved), and GLVs context (MeSa presented alone or MeSa in the background of GLVs) by fitting a logistic regression model with logit link function and binomial error variance in SPlus 6.2 for Windows. The response variable in this analysis was the 
response of an individual predator. The relevance of the explanatory variables was assessed based on the comparison of values of deviance and Akaike's Information Criterion for the models that included combinations of one or more of the three explanatory variables (Agresti 1990; Quinn and Keough 2002). We further tested the fit of the regression model with respect to given explanatory variable by comparing this model with an appropriate reduced model (i.e., a model that contains all terms of the complete model but the explanatory variable tested) by using the log-likelihood ratio test (Agresti 1990). Additionally, we analyzed the effect of the three explanatory variables on the probability of an individual making a choice (i.e., the probability of individual entering any trap vial in the I-tube).

\section{Results}

The first part of this section presents the analysis of the variation detected among the iso-female lines. The second part deals with the analysis of the probability of predators choosing $\mathrm{MeSa}$ using the factors iso-female line, hunger level, and GLVs context. Finally, we discuss the probability of predators making a choice (i.e., the probability of choosing either arm).

Variation among Lines The variation explained by the isofemale line was different from zero $(P=0.02$, Table 1$)$, thus supporting the hypothesis that there is a genetic component in predator responses to MeSa. It is a measure of the total amount of variation among the tested iso-female lines that is due to genetic variation, provided that the differences among the lines due to heterogeneity in rearing conditions are negligible. We detected no systematic change in the average responses of the lines within the period of testing ( $P=0.56$, Table 1$)$. This result supports the conclusion that the observed differences among the lines were not due to systematic differences among the rearing environment of the lines, but rather reflect genetic variation.

In this analysis, the effect of hunger level was significant, but not the GLVs provided as context. However, inspection of the data (Fig. 2; in each panel the ordering of lines from low to high response results in a different sequence of lines) indicates that there is variation in the responses of different lines dependent on the level of these two factors. This interaction was explored further in the second part of the analysis.

Probability of Choosing MeSa We used the logistic regression method to analyze the probability of predators choosing $\mathrm{MeSa}$ in relation to the factors: iso-female line, hunger level, and GLVs as context. Comparison of deviance and AIC values throughout all models (that included combinations of one or more of the three effects) revealed that iso-female line, hunger level, and GLVs as context, all affected the probability that a predator would choose $\mathrm{MeSa}$ (log-likelihood ratio test, $\left.P_{\text {line }}<0.001, P_{\text {hunger }}<0.001, P_{\mathrm{GLVs}}=0.02\right)$. Figure 2 shows the variation among iso-female lines in the proportion of individuals choosing $\mathrm{MeSa}$, categorized by hunger level, when MeSa is presented alone and when it is presented with the background of GLVs. Visual inspection of these figures indicates an interaction between iso-female line, hunger level, and GLVs as context. Therefore, we also compared the deviances and AIC values of the models that included the effects as well as their interaction terms. The final model that best described predator responses included the interaction between the iso-female line and the two fixed effects $(\log$ likelihood ratio tests; $P_{\text {line x hunger }}<0.001, P_{\text {line x GLVs }}<0.001$, $P_{\text {hunger x GLVs }}=0.41, P_{\text {line x hunger x GLVs }}<0.001$ ). Figure 3 exemplifies the three-way interaction by presenting the responses of some of the iso-female lines.

Probability of Making a Choice We constructed a logistic regression model describing the probability that a predator
Table 1 Mixed-effects model of predator responses to MeSa; isofemale line was the random effect and the fixed effects included the hunger level (2 levels: satiated, starved) and GLVs context (2 levels: $\mathrm{MeSa}$ presented alone, MeSa in the background of GLVs). The number of days from the onset of testing the line within a given treatment was added as covariate. The significance of fixed effects, their interaction and the significance of the random effect (i.e., the hypothesis that the variance of random effect $\neq 0$ ) was tested using the likelihood ratio tests (Pinheiro and Bates 2000; Venables and Ripley 2002)

\begin{tabular}{llcc}
\hline Effect & & Likelihood ratio $\left(\mathrm{df}_{1}, \mathrm{df}_{2}\right)$ & $P$ value \\
\hline Random & Iso-female line & $5.25(7,6)$ & 0.02 \\
& Residual & & 0.003 \\
Fixed & Hunger level & $6.75(6,5)$ & 0.05 \\
& GLVs context & $0.05(6,5)$ & 0.009 \\
& Days from onset of testing & $0.34(6,5)$ & 0.56 \\
Interaction & Hunger level: GLVs context & $0.03(7,6)$ & 0.86 \\
\hline
\end{tabular}



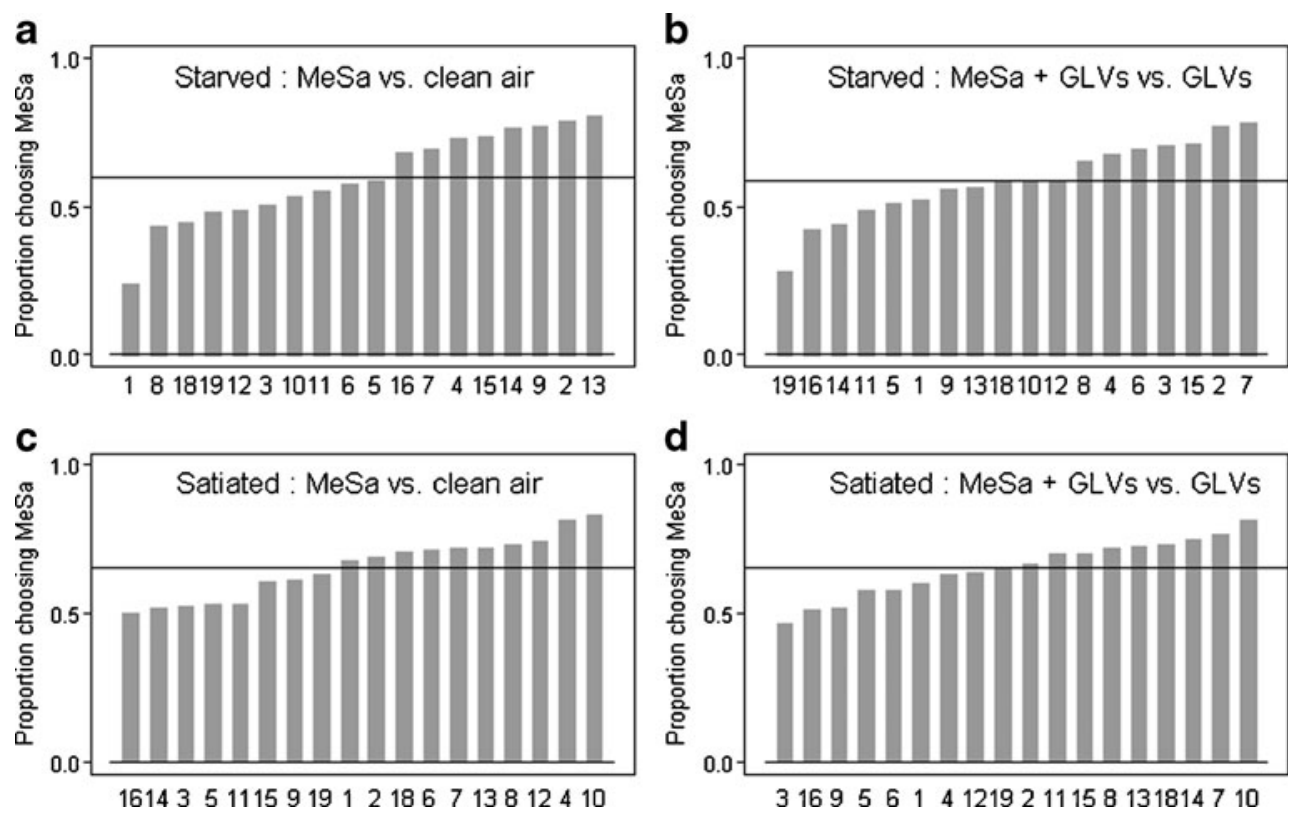

Fig. 2 The responses of eighteen iso-female lines of Phytoseiulus persimilis by starved predators in the tests (a) MeSa plus clean air vs. clean air, (b) MeSa plus the background of GLVs vs. GLVs, and by satiated predators in the tests (c) MeSa plus clean air vs. clean air, (d) MeSa plus the background of GLVs vs. GLVs. The bars show the proportion of individuals choosing the arm containing MeSa (y-axis)

calculated for each line (x-axis). The horizontal lines indicate the average responses obtained by pooling replicates over all iso-female lines within each of the experimental treatments. Note that in each panel the ordering of lines from low to high response results in a different sequence of lines, e.g., line 10 is on the right in (c) and (d) but on the left in (a) and in the middle in (b)

will make a choice in the olfaction test (i.e., it will choose either arm). The full model (i.e., line + test + hunger) explained the responses better than the null model (loglikelihood ratio, $P<0.001)$. All main effects were individually significant $\left(P_{\text {line }}<0.001, P_{\text {test }}<0.001, P_{\text {hunger }}=0.03\right.$, see Fig. 4).

\section{Discussion}

We asked whether there is genetic variation in responses of P. persimilis to the plant volatile $\mathrm{MeSa}$ in a field-collected population. To this end we employed the iso-female line technique where the variation among iso-female lines is

Fig. 3 Illustration of the interaction between iso-female line, hunger level and GLVs context in selected iso-female lines; the graphs present the mean proportions of individuals choosing $\mathrm{MeSa}$ (values on the y-axis) categorized by the factors. Open circles connected by dashed line refer to the responses of the starved predators, closed squares connected by solid line to the responses of the satiated predators, and the $\mathrm{x}$-axis presents the categorization with respect to GLVs context
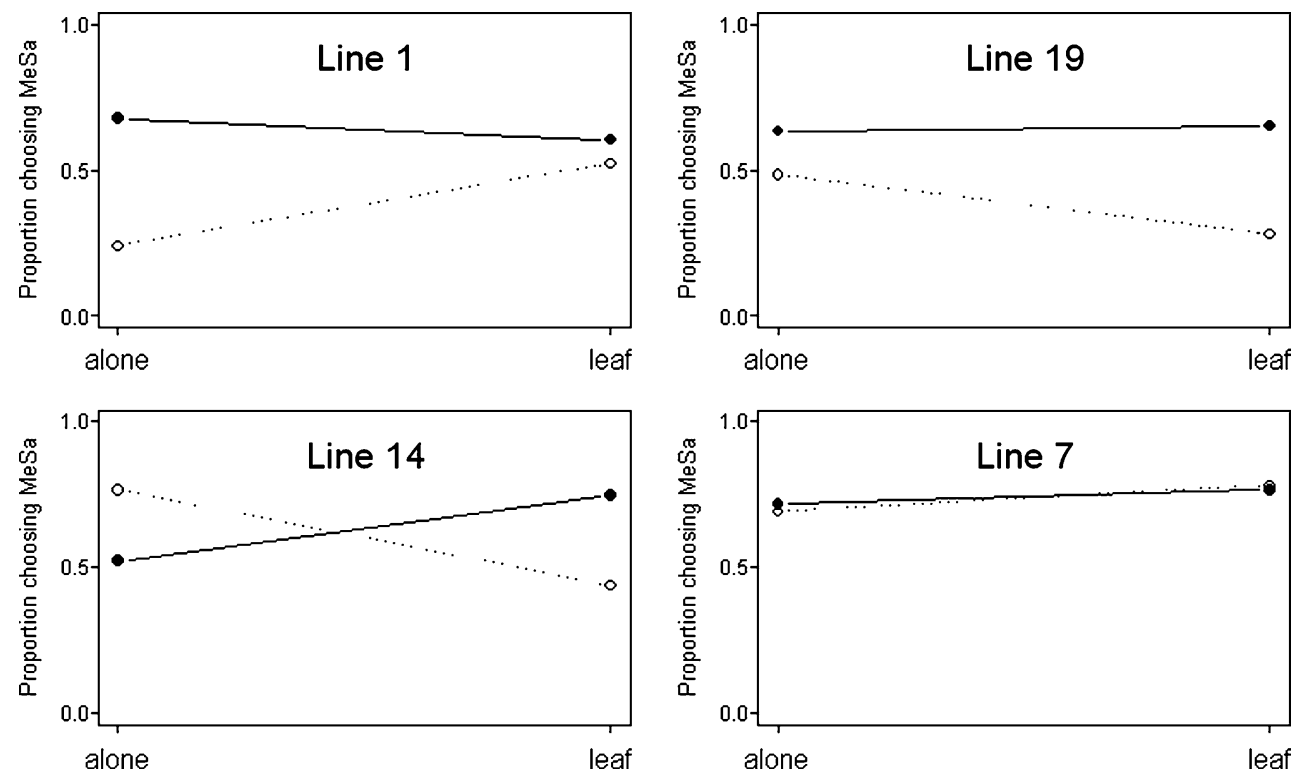
a

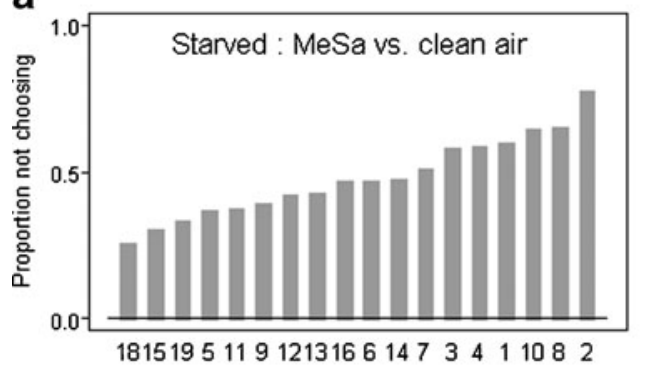

C

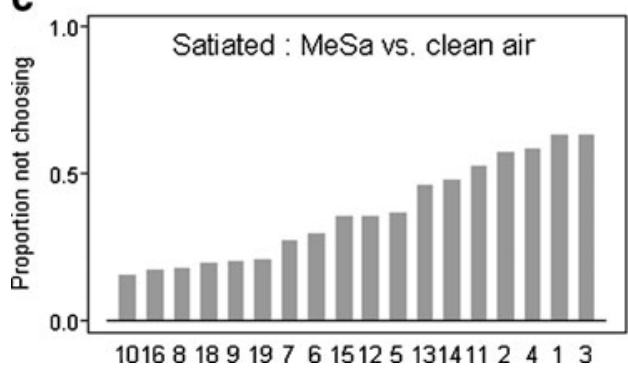

Fig. 4 Variation in the mean proportion of no-choice individuals (yaxis) in the 18 iso-female lines (x-axis) of Phytoseiulus persimilis categorized by hunger level and GLVs context. No-choice individuals are those that remained in the glass tube for the duration of the test, i.e. did not make a choice. The panels present the proportions

interpreted as the variance due to differences in their genes provided the heterogeneity among the rearing environments does not systematically influence the behavior of individual lines. We showed that the variation in predator responses to MeSa explained by the iso-female line was significantly different from zero. From this result, we infer that the studied population harbors genetic variation in olfactory responses to MeSa. There is more variation among the lines when the predators are starved, yet variation is also present in satiated mites. However, it is only when predators are starved that we observe the full range of responses ranging from avoidance to preference. This pattern occurs when predators have a choice of both MeSa presented alone and MeSa against a background of GLVs.

Using the variances quantified from the mixed-effects model for response to MeSa (see Statistical Analysis) we were able to obtain an estimate of broad-sense heritability defined as $\mathrm{H}^{2}=\frac{\sigma^{2} \text { line }}{\sigma^{2} \text { line }+\sigma^{2} \text { residual }}=0.05$, where $\sigma_{\text {residual }}^{2}$ is the remaining amount of unexplained variation that represents the environmental variance (Table 1). The value of estimated broad-sense heritability is relatively small and comparable with most values of narrow-sense heritability of fitness traits (Houle 1992; Visscher et al. 2008; Tien et al. 2009). Moreover, our results indicate that any estimate of heritability may be deflated due to large values of residual, i.e., unexplained, variance detected in this study (Table 1). The residual variance represents the environmental variance, and its magnitude indicates heterogeneity in b

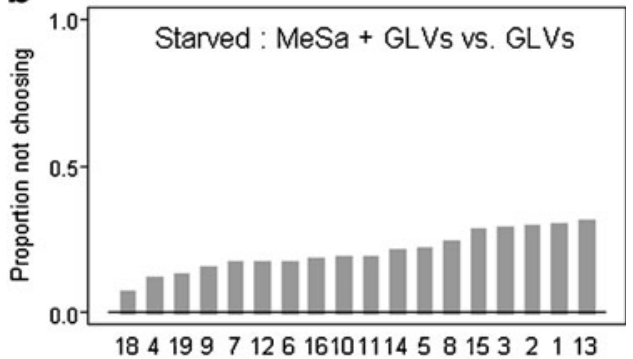

d

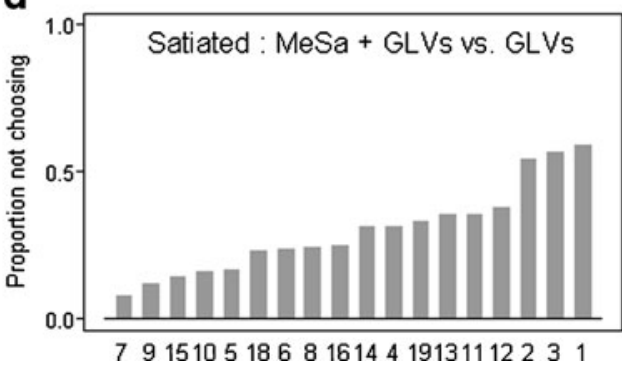

observed for starved predators in the tests (a) MeSa plus clean air vs. clean air, (b) MeSa plus the background of GLVs vs. GLVs, and for satiated predators in tests (c) MeSa plus clean air vs. clean air, (d) MeSa plus the background of GLVs vs. GLVs

other uncontrolled factors, such as predator age, and correlated with it the amount of experience.

To date, studies that have measured the attractiveness of $\mathrm{MeSa}$ (or other herbivore-induced plant volatiles) to natural enemies have not controlled for genetic background (e.g., de Boer and Dicke 2004b; van Wijk et al. 2008). However our results show that predator populations may be genetically variable for the tested responses, and that the behavior of various genotypes may influence the average response measured at the population level. Thus, the average population response may be characterized by a weak attraction or no clear preference if genotypes of extreme innate preferences are tested together and their responses pooled. To illustrate this, we calculated the proportions of individuals choosing MeSa by pooling the replicates over all iso-female lines for each of the experimental treatments. These values (shown in Fig. 2 as horizontal lines) may be treated as estimates of the responses to MeSa measured at the level of the base population from which the lines originated, under the assumption that different iso-female lines (i.e., genotypes) are represented. Indeed, there is little difference among the experimental treatments.

The inclusion of GLVs in the background of MeSa presentation does not, at first glance, appear to change the overall result that a wider range of responses is expressed by starved predators (compare Fig. 2a, c and Fig. 2b, d). However, closer inspection reveals that the addition of the 
background of GLVs does change the behavior of individual lines. We observed a whole range of patterns in the responses of the lines that exemplify a significant three-way interaction between iso-female line, hunger level, and GLVs as background (except for line 5 that shows indifferent responses in all tests without exception). In particular, there are iso-female lines characterized by an extreme response to MeSa if the predators are starved and the choice-test involves MeSa in the background of GLVs, whereas their responses in all the other treatments are indifferent (lines 19 and 3). The example of these lines may indicate that testing the responses of starved predators to MeSa presented in the background of GLVs are essential conditions for eliciting an innate response of the predators. However, there are also lines characterized by extreme responses to $\mathrm{MeSa}$ if the predators are starved and the choice-test involves MeSa presented alone (e.g., lines 1 and 9), and predator responses in all the other treatments are indifferent. The example of these lines may thus indicate that the adding of GLVs as a background to MeSa confounds the predator's perception of this volatile. The remaining iso-female lines show yet other patterns of interaction among the factors iso-female line, hunger level, and GLVs as background (see Fig. 3 for examples).

We conclude therefore that the responses of $P$. persimilis to MeSa are context-dependent in that they depend on the genetically determined preference of the tested individuals (i.e., their genotypes) as well as on the environmental context in which the volatile is presented. Further research is needed to understand how a biologically relevant single compound cue is perceived and interpreted when presented in a mixture (van Wijk 2007; Shröder and Hilker 2008), a more realistic situation in nature. In our study, the addition of a background odor of GLVs increased the proportion of individuals making a choice in olfaction tests (Fig. 4). Thus, we conclude that GLVs affected the predator's perception of MeSa. This effect of GLVs on the willingness to choose varied among individual lines (which indicates a genetic basis also in this aspect of behavior).

Given the genetic basis in the responses to MeSa in this population collected from the field, we conclude that there is opportunity for an evolutionary response on the part of the predators to using $\mathrm{MeSa}$ as a plant signal indicating prey presence. The distribution of prey is patchy and unpredictable under natural conditions. Hence, it is expected that $P$. persimilis undergoes long-lasting food deprivation during dispersal (Sabelis and Afman 1994). Our findings may indicate stronger reliance of the predator on genetic predispositions during this migratory phase in which the predator alternates passive aerial dispersal and local exploration for prey patches. Thus, natural selection may be more efficient in shaping the olfactory responses when the predators are starved and therefore probably migrating.
Our results show that the responses of $P$. persimilis to plant volatiles are far from simple. Even in the case of a single plant volatile, we observed genetic variation expressed in a context-dependent manner. Iso-female lines created from a field-collected base population showed responses to MeSa ranging from preference to avoidance (although the latter response was much less common). The variation is still present in the population and conflicts with the prediction that preference for environments associated with the presence of MeSa has fitness benefits (i.e. helps locate the prey) and should therefore prevail. This raises the question of the mechanism that maintains the observed genetic variation in the field; the question could be addressed with selection experiments for olfactory responses to single volatile compounds and mixtures. Our results suggest that such experiments will be more successful if conducted on starved predators.

Open Access This article is distributed under the terms of the Creative Commons Attribution Noncommercial License which permits any noncommercial use, distribution, and reproduction in any medium, provided the original author(s) and source are credited.

\section{References}

Agresti, A. 1990. Categorical Data Analysis. Wiley, New York.

Allison, J. D., and HARE, J. D. 2009. Learned and naïve natural enemy responses and the interpretation of volatile organic compounds as cue or signals. New Phytol. 184:768-782.

Ament, K., Krasikov, V., Allmann, S., Rep, M., Takken, F. L. W., and SchUURINK, R. C. 2010. Methyl salicylate production in tomato affects biotic interactions. Plant J. 62:124-134.

David, J. R., Gibert, P., Legout, H., PÉtavy, G., CAPy, P., and MoreteaU, B. 2005. Isofemale lines in Drosophia: an empirical approach to quantitative trait analysis in natural populations. Heredity 94:3-12.

De Boer, J. G., and DiCKE, M. 2004a. Experience with methyl salicylate affects behavioural responses of a predatory mite to blends of herbivore-induced plant volatiles. Entomol. Exp. Appl. 110:181-189.

De BoER, J. G., and DicKe, M. 2004b. The role of methyl salicylate in prey searching behaviour of the predatory mite Phytoseiulus persimilis. J. Chem. Ecol. 30:255-271.

De Boer, J. G., Posthumus, M. A., and Dicke, M. 2004. Identification of volatiles that are used in discrimination between plants infested with prey or nonprey herbivores by a predatory mite. J. Chem. Ecol. 30:2215-2230.

De Moraes, G. J., Mcmurtry, J. A., Denmark, H. A., and Campos, C. B. 2004. A revised catalog of the mite family Phytoseiidae. Zootaxa 434:494.

Dicke, M., and SABELIS, M. W. 1988. How plants obtain predatory mites as bodyguards. Neth. J. Zool. 38:148-165.

DiCKE, M., and VAN LOON, J. J. A. 2000. Multitrophic effects of herbivore-induced plant volatiles in an evolutionary context. Entomol Exp. Appl. 97:237-249.

Dicke, M., Van Beek, T. A., Posthumus, M. A., Dom, N. B., Van BoKhoven, H., and DE GroOT, A. E. 1990. Isolation and identification of volatile kairomone that affects acarine predator- 
prey interactions: Involvement of host plant in its production. $J$. Chem. Ecol. 16:381-396.

Dicke, M., Gols, R., Ludeking, D., and Posthumus, M. A. 1999. Jasmonic acid and herbivory differentially induce carnivoreattracting plant volatiles in lima bean plants. J. Chem. Ecol. 25:1907-1922.

HATANAKA, A. 1993. The biogeneration of green odors by green leaves. Phytochemistry 34:1201-1218.

Helle, W., Bolland, H. R., Van Arendonk, R., De Boer, R., Schulten, G. G. M., and Russell, V. M. 1978. Genetic evidence for biparental males in haplo-diploid predator mites (Acaria: Phytoseiidae). Genetica 49:165-171.

HouLE, D. 1992. Comparing evolvability and variability of quantitative traits. Genetics 130:195-204.

JANSSEN, A., SABelis, M. W., and BRUIN, J. 2002. Evolution of herbivore-induced plant volatiles. Oikos 97:134-138.

Jia, F., Margolies, D. C., Boyer, J. E., and Charlton, R. E. 2002. Genetic variation among foraging traits in inbred lines of a predatory mites. Heredity $88: 371-379$.

KESSLER, A., and BALDwIN, I. T. 2001. Defensive function of herbivore-induced plant volatile emissions in nature. Science 291:2141-2144

Margolies, D. C., Dabelis, M. W., and Boyer, J. E. 1997. Response of a phytoseiid predator to herbivore-induced plant volatiles: selection on attraction and effect on prey exploitation. J. Insect Behav. 10:695-709.

Matsui, K., Kurishita, S., Hisamitsu, A., and KaJiwara, T. 2000. A lipid-hydrolysing activity involved in hexenal formation. Biochem. Soc. Trans. 28:857-860.

Nelson-Rees, W. A., Hoy, M. A., and Rosh, R. T. 1980. Heterochromatinization, chromatine elimination and haploidization in the parahaploid mite Metaseiulus occidentalis (Nesbitt) (Acarina: Phytoseiidae). Chromosoma 77:263-276.

PELS, B., and SABELIS, M. W. 1999. Local dynamics, overexploitation and predator dispersal in an acarine predator-prey system. Oikos 86:573-583.

Pinheiro, J. C., and Bates, D. M., 2000. Mixed-Effects Models in S and S-PLUS. Statistics and Computing Series. Springer, New York.

QuinN, G. P., and KeOUgh, M. J. 2002. Experimental Design and Data Analysis for Biologists. Cambridge University Press, Cambridge.

SABELIS, M. W. 1981. Biological control of two-spotted spidermites using phytoseiid predators. Agricultural Research Reports 910 Pudoc, Wageningen.

Sabelis, M. W., and Afman, B. P. 1994. Synomone-induced suppression of take-off in the phytoseiid mite, Phytoseiulus persimilis. Entomol Exp. Appl. 18:711-721.

SABELIS, M. W., and VAN DE BAAN, H. E. 1983. Location of distant spider-mite colonies by phytoseiid predators: demonstration of specific kairomones emitted by Tetranychus urticae and Panonychus ulmi (Acari: Phytoseiidae, Tetranychidae). Entomol. Exp. Appl. 33:303-314.

Sabelis, M. W., and Van Der MeER, J. 1986. Local dynamics of the interaction between predatory mites and the to-spotted spider mites, pp. 233-244, in J. A. J. Metz and O. Diekmann (eds.). Dynamics of Physiologically Structured Populations, Lecture Notes in Bioinformatics 68. Springer-Verlag, Berlin.

SABELIS, M. W., and VAN DeR WeEL, J. J. 1993. Anemotactic responses of the predatory mite, Phytoseiulus persimilis Athias-Heniot, and their role in prey finding. Exp. Appl. Acarol. 17:521-529.

Sabelis, M. W., VermaAt, J. E., and Groenveld, A. 1984. Arrestment responses of the predatory mite, Phytoseiulus persimilis, to steep odour gradients of kairomones. Physiol. Entomol. 9:437-446.

Sabelis, M. W., Takabayashi, J., Janssen, A., Kant, M. R., VAN WiJK, M., Sznajder, B., Aratchige, N. S., LeSnA, I., Belluire, B., and SCHUURINK, R. C. 2007. Ecology meets plant physiology: Herbivore-induced plant responses and their indirect effects on arthropod communities, pp. 188-217, in T. Oghushi, T. P. Craig and P. W. Price (eds.). Ecological Communities: Plant Mediation in Indirect Interaction Webs. Cambridge University Press, Cambridge.

SHRÖDER, R., and HILKER, M. 2008. The relevance of background odor in resource location by insects: a behavioural approach. Bioscience 58:308-316.

TIEN, N. S. H., SABELIS, M. W., and EgAS, M. 2009. The heritability of defense and life-history traits in the two-spotted spider mite. Evol. Ecol. Res. 11:1271-1281.

Van Den Boom, C. E. M., Van Beek, T. A., Posthumus, M. A., De Groot, Æ., and DiCKE, M. 2004. Qualitative and quantitative variation between volatile profiles induced by Tetranychus urticae feeding on different plants of various families. J. Chem. Ecol. 30:69-89.

Van Der Meijden E., and Klinkhamer, P. G. L. 2000. Conflicting interest of plants and the natural enemies of herbivores. Oikos 89:202-208

VAN WIJK, M. 2007. Deciphering the code of herbivore-induced plant odours: the whole is different from the sum of its parts. PhD dissertation. University of Amsterdam.

VAN WiJK, M., De BRUiJn, P. J. A., and SABelis, M. W. 2008. Predator attraction to herbivore induced plant odours is not a consequence of attraction to individual herbivore-induced plant volatiles. J. Chem. Ecol. 32:1573-1561.

Venables, W. N., and Ripley, B. D. 2002. Modern Applied Statistics with S. 4th edn. Springer.

VISSCHER, P.M., HILL, W.G., and WrAY, N.R. 2008. Heritability in the genomics era - concepts and misconceptions. Nature Rev. Genet. 9:255-266. 